\title{
Computational Algorithm for Covariant Series Expansions in General Relativity
}

\author{
Ivan Potashov ${ }^{1, \star}$ and Alexander Tsirulev ${ }^{1, \star \star}$ \\ ${ }^{1}$ Faculty of Mathematics, Tver State University, Sadovyi per. 35, Tver, Russia, 170002
}

\begin{abstract}
We present a new algorithm for computing covariant power expansions of tensor fields in generalized Riemannian normal coordinates, introduced in some neighborhood of a parallelized $k$-dimensional submanifold $(k=0,1, \ldots<n$; the case $k=0$ corresponds to a point), by transforming the expansions to the corresponding Taylor series. For an arbitrary real analytic tensor field, the coefficients of such series are expressed in terms of its covariant derivatives and covariant derivatives of the curvature and the torsion. The algorithm computes the corresponding Taylor polynomials of arbitrary orders for the field components and is applicable to connections that are, in general, nonmetric and not torsion-free. We show that this computational problem belongs to the complexity class LEXP.
\end{abstract}

\section{Introduction}

Covariant series expansions in Riemann normal coordinates [1, 2], in Fermi normal coordinates [3, 4], and in some other geodesic coordinate systems (e.g., see Ref. [5]) find important applications in general relativity [6-9]). Explicit formulas and recipes for coefficients of the series are given in Refs. [10-15], so that, from a formal point of view, the problem is solved completely. However, the complexity of calculations increases rapidly with increasing the order of a covariant expansion and there obviously arises the question whether we can organize these calculations on a computer. To avoid confusion, it is essential to note that the problem, in the context of calculating a covariant series, can be set in different ways depending on the explicit form of the expansion. We employ the mathematical framework of Ref. [15] where the problem is considered with a sufficient degree of generality: one needs only a connection to form the series; the connection is not assumed to be metric or torsion-free; the covariant series are defined in some normal neighborhood of an arbitrary submanifold. In our setting, having established a connection (or spacetime metric), one needs to compute the covariant derivatives of the curvature (and, in general, of the torsion), to collect like terms, and then to compute coefficients of coordinate monomials.

Sec. 2 contains some necessary mathematical preliminaries and a short description of the computational algorithm. In Sec. 3 we make the estimations of numbers of multiplications at various stages of the algorithm and show that the complexity class of the algorithm is LEXP.

${ }^{\star}$ e-mail: potashov.im@tversu.ru

$\star \star$ e-mail: tsirulev.an@tversu.ru 


\section{General expansions and computational algorithm}

We deal with a real analytic manifold $H, \operatorname{dim} H=n+m, n>m \geqslant 0$, and a connected parallelizable submanifold $M \subset H, \operatorname{dim} M=m ; M$ is a point if $m=0$. At each point $p \in M$ the tangent space $T_{p}(H)$ splits into the direct sum $T_{p}(M) \oplus N_{p}$, where the subspace $N_{p}$ is normal (transverse, if the metric is not specified) to $M$. Let $\left\{\mathrm{e}_{\alpha}\right\}$ and $\left\{\mathrm{e}_{a}\right\}$ be bases for $N_{p}$ and $T_{p}(M)$ respectively, so that their union is a basis for $T_{p}(H)$. We assume that the Latin indices $i, j, k, l$ take the values $1, \ldots, n+m$, while the Greek indices $\alpha, \beta, \gamma$ run from 1 to $n$, and the Latin indices $a, b$ run from $m+1$ to $n+m$; they are used to denote tensor components in $T_{p}(H), N_{p}$, and $T_{p}(M)$ respectively. For these indices, we also adopt Einstein's summation convention, that is, a pair of identical indices, with one lower index and one upper index, denotes summation over their range. Let $\nabla$ be a linear connection on $H$ and $\nabla_{\mathrm{e}_{m}} \mathrm{e}_{k}=\Gamma_{k m}^{i} \mathrm{e}_{i}, \nabla_{\mathrm{e}_{m}} X=X_{; m}^{k} \mathrm{e}_{k} ; R_{m i j}^{k}=\left\langle\mathrm{e}^{k}, \nabla_{i} \nabla_{j} \mathrm{e}_{m}-\nabla_{j} \nabla_{i} \mathrm{e}_{m}-\nabla_{\left[\mathrm{e}_{i}, \mathrm{e}_{j}\right]} \mathrm{e}_{m}\right\rangle$ are the curvature components. In what follows, for the sake of shortness, we restrict our consideration to the case of torsion-free connections.

If $\gamma_{X}$ is the geodesic in the direction of a vector $X=X^{\alpha} \mathrm{e}_{\alpha} \in N_{p}$, such that $\gamma_{X}(0)=p, \gamma_{X}(1)=q \in H$, then $X^{1}, \ldots, X^{n}$ are normal coordinates of the point $q$ (the $\exp$ map). Now let $Q$ be a tensor field of type $(r, s)$ (of type $(0,2)$, in the particular case of a metric $g$ ) in a normal neighborhood of the submanifold $M$, then the covariant series expansion for $Q$ has the form [15]

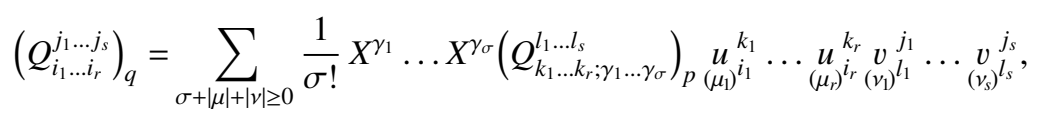

where $\sigma, \mu_{1}, \ldots, \mu_{r}, v_{1}, \ldots, v_{s} \geq 0, \quad|\mu|=\sum_{t=1}^{r} \mu_{t}, \quad|v|=\sum_{t=1}^{s} v_{t}, \underset{(0)}{u_{i}^{k}}=\underset{(0)}{v i}{ }^{k}=\delta_{i}^{k}, \quad \underset{(1)}{u_{\alpha}^{k}}=0, \quad \underset{(1)}{u_{a}^{k}}=X^{\beta}\left(\Gamma_{\beta a}^{k}\right)_{p}$, $\underset{(\mu)}{u_{i}^{k}}=\frac{1}{\mu(\mu+\varepsilon(i))} \sum_{\sigma=2}^{\mu} \frac{1}{(\sigma-2) !} X^{\alpha_{1}} \ldots X^{\alpha_{\sigma}}\left(R_{\alpha_{1} \alpha_{2} l ; \alpha_{3} \ldots \alpha_{\sigma}}^{k}\right)_{p} \underset{(\mu-\sigma)_{i}}{u^{l}}(\mu \geq 2), \quad \underset{(\mu)^{i}}{v^{k}}=-\sum_{\sigma=1}^{\mu} \underset{(\mu-\sigma)^{l}}{v_{(\sigma)}^{k}} u_{i}^{l}(\mu \geq 1)$,

$\varepsilon(\alpha)=1$ and $\varepsilon(a)=-1$ in the ranges of $\alpha$ and $a$. We come now to the problem of algorithmic reducibility of the expansion (1). The number of coordinate monomials in (1) is equal to the number of terms of the corresponding Taylor series. The most difficult step is the computation of the coefficients of monomials in matrices $u_{j}^{i}$; if it has been done, the monomials in (1) can be isolated by well-known methods. Therefore, we restrict the discussion to these computations.

For each $\mu \geq 2$ we generate the lexicographically ordered set of the sequences of $n$ whole numbers

$$
S_{\mu}=\left\{\left(A_{1}, \ldots, A_{n}\right) \mid A_{1}+\cdots+A_{n}=\mu\right\}=\left\{S_{\mu}[1], \ldots, S_{\mu}[N]\right\}, \quad N=\left|S_{\mu}\right|=\left(\begin{array}{c}
n+\mu-1 \\
\mu-1
\end{array}\right),
$$

and for each element $S_{\mu}[p]=\left(A_{1}, \ldots, A_{n}\right) \in S_{\mu}, 1 \leq p \leq N$, we consider the set

$$
S_{\mu}^{p}=\left\{\left(\alpha_{1}, \ldots, \alpha_{\mu}\right) \in\{1,2, \ldots, n\}^{\mu}\right\}=\left\{S_{\mu}^{p}[1], \ldots, S_{\mu}^{p}[M]\right\}, \quad M=\left|S_{\mu}^{p}\right|=\frac{\mu !}{A_{1} ! A_{2} ! \ldots A_{n} !},
$$

the elements of which (sequences of length $\mu$ ) are the permutations of the sequence which consists of $A_{1}$ numbers $1, A_{2}$ numbers $2, \ldots, A_{n}$ numbers $n$. We also need to form the set $C_{\mu}$ of the sequences of variable length and the collection of the corresponding rational constants $h: C_{\mu} \rightarrow \mathbb{Q}$ :

$$
\begin{gathered}
C_{\mu}=\left\{\left(\sigma_{1}, \ldots, \sigma_{\tau}\right) \mid \sigma_{1}+\cdots+\sigma_{\tau}=\mu, \sigma_{1}, \ldots, \sigma_{\tau} \geqslant 2,1 \leqslant \tau \leqslant[\mu / 2]\right\}, \quad N_{1}=\left|C_{\mu}\right|=\sum_{\tau=1}^{[\mu / 2]}\left(\begin{array}{c}
\mu-\tau-1 \\
\tau-1
\end{array}\right), \\
h\left(\sigma_{1}, \ldots, \sigma_{\tau}\right)=\prod_{r=1}^{\tau} \frac{\xi\left(\sigma_{r}+\sigma_{r+1}+\ldots+\sigma_{\tau}\right)}{\left(\sigma_{r}-2\right) !}, \quad \xi(\sigma)=\frac{1}{\sigma(\sigma+1)} .
\end{gathered}
$$




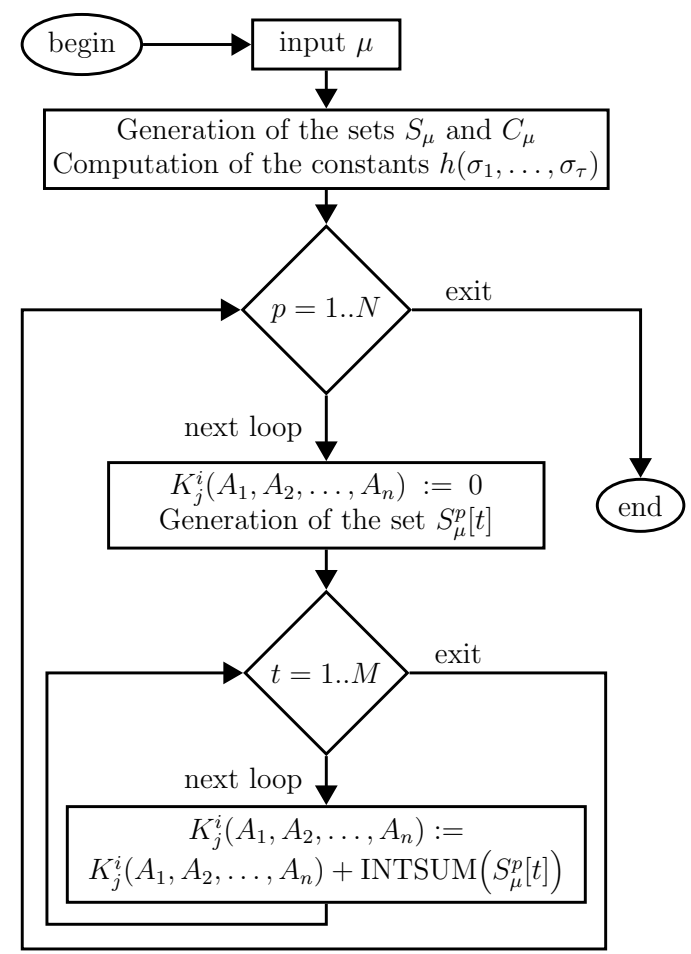

Figure 1. Block diagram of the algorithm

For the matrix $u_{i}^{j}$ of order $\mu$, we have

$$
\underset{(\mu)}{u_{j}^{i}}=\sum_{S_{\mu}} K_{j}^{i}\left(A_{1}, \ldots, A_{n}\right)\left(X^{1}\right)^{A_{1}} \ldots\left(X^{n}\right)^{A_{n}}
$$

where all the $K_{j}^{i}\left(A_{1}, \ldots, A_{n}\right)$ should be expressed in terms of the curvature. The algorithm is shown schematically in Fig. 1 . We compute $K_{j}^{i}$ for the sequence $S_{\mu}[p]=\left(A_{1}, \ldots, A_{n}\right)$ at the $p^{\text {th }}$ step of the external loop, where $p$ runs from 1 to $N$. In doing this we generate the set $C_{\mu}$ and compute, for each of its elements $\left(\sigma_{1}, \ldots, \sigma_{\tau}\right)$, the constants (4). The covariant derivatives for $S_{\mu}^{p}$ are computed by a special iterative procedure. Let $n=3, \mu=4$, and $S_{4}[7]=(1,2,1)$. Thus $C_{\mu}=\{(4),(2,2)\}$ and, e.g., if $\left(\alpha_{1}, \alpha_{2}, \alpha_{3}, \alpha_{4}\right)=(2,1,2,3)$, the procedure gives explicit expressions for $R_{21 j ; 23}^{i}, R_{21 j}^{i}$, and $R_{23 j}^{k}$. In the internal loop, the algorithm calls the special procedure INTSUM. Finally, for the coefficients in (5) we obtain

$$
K_{j}^{i}\left(A_{1}, \ldots, A_{n}\right)=\sum_{t=1}^{M} \operatorname{INTSUM}\left(S_{\mu}^{p}[t]\right)
$$

$$
\operatorname{INTSUM}\left(S_{\mu}^{p}[t]\right)=\sum_{C_{\mu}} h\left(\sigma_{1}, \ldots, \sigma_{\tau}\right) R_{\alpha_{1} \alpha_{2} l_{1} ; \alpha_{3} \ldots \alpha_{\delta_{1}}}^{i} R_{\alpha_{\delta_{1}+1} \alpha_{\delta_{1}+2} l_{2} ; \alpha_{\delta_{1}+3} \ldots \alpha_{\delta_{2}}}^{l_{1}} \ldots R_{\alpha_{\delta_{\tau-1}+1} \alpha_{\delta_{\tau-1}+2} j ; \alpha_{\delta_{\tau-1}+3} \ldots \alpha_{\delta_{\tau}}}^{l_{\tau-1}}
$$

where $S_{\mu}^{p}[t]=\left(\alpha_{1}, \ldots, \alpha_{\mu}\right)$ and $\delta_{r} \equiv \sum_{s=1}^{r} \sigma_{s}$. 


\section{Computational complexity of the algorithm}

There are two ways of summation in the formula (6) for $K_{j}^{i}\left(A_{1}, \ldots, A_{n}\right)$ : one is over the set $S_{\mu}^{p}$ and the other is over the set $C_{\mu}$ in the procedure INTSUM. In addition, each summand in the latter sum is a matrix product with $\tau-1$ summation indices; it gives $(n+m)^{\tau-1}$ multiplications. The total number of multiplications in INTSUM is the sum of $N_{1}$ multiplications by the factors $h$ and the number $N_{2}$ of matrix multiplications. From (2) and (3), using the generating functions for the sums (particularly the Binet's Fibonacci number formula), we find

$$
\begin{gathered}
N_{1}=\sum_{\tau=0}^{[\mu / 2]-1}\left(\begin{array}{c}
\mu-\tau-2 \\
\tau
\end{array}\right)=F_{\mu-1}=\frac{1}{\sqrt{5}}\left[\left(\frac{1+\sqrt{5}}{2}\right)^{\mu-1}-\left(\frac{1-\sqrt{5}}{2}\right)^{\mu-1}\right] \leqslant \frac{1}{\sqrt{5}}\left(\frac{1+\sqrt{1+4(n+m)}}{2}\right)^{\mu-1}, \\
N_{2}+1=\sum_{\tau=1}^{[\mu / 2]}\left(\begin{array}{c}
\mu-\tau-1 \\
\tau-1
\end{array}\right)(n+m)^{\tau-1}=\frac{(1+\sqrt{1+4(n+m)})^{\mu-1}}{2^{\mu-1} \sqrt{1+4 n+4 m}}-\frac{(1-\sqrt{1+4(n+m)})^{\mu-1}}{2^{\mu-1} \sqrt{1+4 n+4 m}} .
\end{gathered}
$$

From here we obtain the estimation

$$
N_{1}+N_{2}=O\left(\left(\frac{1+\sqrt{1+4(n+m)}}{2}\right)^{\mu}\right), \mu \rightarrow \infty, n+m \text { is fixed. }
$$

For the number of multiplications on the right hand side in formula (6) and the total number of multiplications in computing all the coefficient $K_{j}^{i}\left(A_{1}, \ldots, A_{n}\right)$ we have, respectively, the estimations

$$
\left(N_{1}+N_{2}\right) \cdot M=\left(N_{1}+N_{2}\right) \cdot \frac{\mu !}{A_{1} ! \ldots A_{n} !}, \quad\left(N_{1}+N_{2}\right) \cdot \sum_{\substack{A_{1}+\cdots+A_{n}=\mu \\ A_{1}, \ldots, A_{n} \geqslant 0}} \frac{\mu !}{A_{1} ! \ldots A_{n} !}=\left(N_{1}+N_{2}\right) \cdot n^{\mu}
$$

Finally, we have $O\left(\frac{n+n \sqrt{1+4(n+m)}}{2}\right)^{\mu}$ for the estimation of computational complexity of the algorithm. The algorithm belongs to the complexity class $L E X P \equiv E \subset$ EXPTIME.

\section{References}

[1] L.P. Eisenhart, Riemannian Geometry (Princeton University Press, Princeton, 1926)

[2] A.Z. Petrov, Einstein Spaces (Pergamon Press, Oxford, 1969)

[3] F.K. Manasse and G.W. Misner, J. Math. Phys. 4, 735-745 (1963)

[4] E. Poisson, A. Pound and I. Vega, Living Rev. Relativ. 14, 7 (2011)

[5] V. Iyer and R.M. Wald, Phys. Rev. D 50, 846-864, (1994)

[6] D. Bini, A. Geralico and R.T. Jantzen, Gen. Relativ. Grav. 44, 1837-1853 (2011)

[7] A.I. Harte, Class. Quant. Grav. 27, 135002 (2010)

[8] M.N.R. Wohlfarth and C. Pfeifer, Phys. Rev. D 87, 024031 (2013)

[9] K.-P. Marzlin, Gen. Relativ. Grav. 26, 619-636 (1994)

[10] P. Mukhopadhyay, Reviews in Math. Phys. 26, 1, 1350019 (2013)

[11] L. Brewin, Class. Quantum Grav. 26, 175017 (2009)

[12] D. Klein and P. Collas, J. Math. Phys. 51, 022501 (2010)

[13] B.Z. Iliev, Handbook of Normal Frames and Coordinates (Birkhäuser Verlag, Berlin, 2006)

[14] U. Müller, C. Schubert and A.E.M. van de Ven, Gen. Relativ. Grav. 1, 1759-1765 (1999)

[15] A.N. Tsirulev, Theor. Math. Phys. 102, 3, 245-250 (1995) 